\title{
Delay Issues in Linear Sensory Networks ${ }^{1}$
}

\author{
Cédric Florens, Masoud Sharif, and R. J. McEliece \\ Dept. Electrical Engineering \\ California Institute of Technology \\ Pasadena, CA 91125 \\ \{florens, masoud, rjm\}@systems.caltech.edu
}

\begin{abstract}
In this paper, we study the data collection function in sensory networks. Specifically we derive relationships between data collection time and transmission range, data packet size and channel noise in the simple line scenario. To develop intuition these relationships are studied in the limit case where the number of sensor nodes becomes large.
\end{abstract}

A sensor network is a finite collection of $n$ identical nodes $\left\{N_{1}, \ldots, N_{n}\right\}$. Each node $N_{i}$ carries, after an observation period, an integer $\nu_{i}$ number of data packets to be collected by the processing center (BS) $N_{0}$. Nodes (including BS) have a common transmission/interference range $r$. We assume that time is slotted and a one hop transmission consumes one time slot (TS). The network is further assumed to be synchronous. A node can only transmit/receive one data packet per TS. Multiple transmissions may occur within the network in one TS under this interference model [1] by virtue of spatial separation. For a one-sided line network (BS located at one end) of length ${ }^{2} n$ in which the $i$ 'th node has $\nu_{i}$ packets and is equipped with directional antennas, the minimum collection time of the packets at the BS, denoted by $T_{\min }\left(\boldsymbol{\nu}_{\boldsymbol{n}}\right)$, is, assuming $r=1$ hop:

$$
T_{\text {min }}\left(\boldsymbol{\nu}_{\boldsymbol{n}}\right)=\max _{1 \leq i \leq n-1}\left(i-1+\nu_{i}+2 \sum_{j \geq i+1}^{n} \nu_{j}\right)
$$

where $\boldsymbol{\nu}_{\boldsymbol{n}}=\left(\nu_{1}, \ldots, \nu_{n}\right)$.

Furthermore, we can prove that [2]

$$
\lim _{n \rightarrow \infty} \frac{\mathbb{E}\left\{T_{\min }\left(\boldsymbol{\nu}_{\boldsymbol{n}}\right)\right\}}{n}= \begin{cases}2 \mu & \text { if } \mu \geq 1 / 2 \\ 1 & \text { if } \mu \leq 1 / 2\end{cases}
$$

where $\nu_{i}$ 's are i.i.d. random variables $\nu_{i} \in\{0,1, \ldots, m-1\}$ with mean $\mu$ and variance $\sigma^{2}$ where $\mu, \sigma^{2}, m$ are constants independent of $n$.

Note that the minimum collection time in (1) is achieved under the assumption that each sensor node has a perfect knowledge of the network topology and data packets locations. A more practical strategy that does not require knowledge of the packet locations and therefore can be run in a distributed fashion is as follows: nodes at odd (resp. even) distance from the BS transmit to their closest neighbors toward the BS at odd (resp. even) TS. The next theorem analyzes the delay performance of the above decentralized algorithm.

Theorem 1. The collection time of the packets at the BS under the previously described strategy, denoted by $T\left(\boldsymbol{\nu}_{\boldsymbol{n}}\right)$, is:

where $\boldsymbol{\nu}_{\boldsymbol{n}}=\left(\nu_{1}, \ldots, \nu_{n}\right)$.

$$
\begin{aligned}
& T\left(\boldsymbol{\nu}_{\boldsymbol{n}}\right)=\max _{1 \leq i \leq n}\left(2\left\lfloor\frac{i}{2}\right\rfloor-1+2 \sum_{j \geq i}^{n} \nu_{j}\right) \\
& \left(\nu_{1}, \ldots, \nu_{n}\right) .
\end{aligned}
$$

\footnotetext{
${ }^{1}$ This work was supported by the Caltech Lee Center for Advanced Networking.

${ }^{2}$ Implicitly we assume that the distance to the BS of the furthest node carrying a data packet is $n$.
}

Furthermore this strategy is asymptotically optimal, that is presents the asymptotic delay performance as in Eq. (2). The next two theorems study the impact of the transmission range on the minimum data collection time.

Theorem 2. The minimum collection time of the packets at the $B S$ as a function of the transmission range $h$ in hops is:

$$
T_{\text {min }}\left(h, \nu_{n}\right)=\max \left(S^{\prime}, S_{h+1}, \ldots, S_{n-h}\right)
$$

where

$$
\begin{aligned}
S_{i} & =n_{i}+\left\lfloor\frac{n_{i}-1+d \bmod h}{h}\right\rfloor+\left\lfloor\frac{d}{h}\right\rfloor+1 \\
S^{\prime} & =S_{0}+\max \left(\sum_{j=1}^{l} \nu_{j}-1,0\right)+\sum_{j=l+1}^{h} \nu_{j}
\end{aligned}
$$

$n_{i}=\sum_{j>i+h} \nu_{j}$, and $0 \leq l \leq h-1$ such that $l+n_{0}=0$ $\bmod h$.

For $h$ fixed, we can further prove that

$$
\lim _{n \rightarrow \infty} \frac{\mathbb{E}\left\{T_{\min }\left(h, \boldsymbol{\nu}_{\boldsymbol{n}}\right)\right\}}{n}= \begin{cases}\left(1+\frac{1}{h}\right) \mu & \text { if } \mu \geq \frac{1}{h+1} \\ 1+\frac{1}{h} & \text { if } \mu \leq \frac{1}{h+1}\end{cases}
$$

Next we examine the potential gain in time obtained by splitting packets into subpackets.

Theorem 3. Given a line network $\boldsymbol{\nu}_{\boldsymbol{n}}$ the minimum data collection time may be reduced by a factor $G\left(\boldsymbol{\nu}_{n}, k\right), k \geq G \geq 1$ by splitting the data packets into $k$ sub-packets and reducing the length of a TS accordingly. $G\left(\boldsymbol{\nu}_{n}, k\right)$ is a non-decreasing function of $k$ and the maximum achievable gain is:

$G_{\text {max }}\left(\boldsymbol{\nu}_{\boldsymbol{n}}\right)=\lim _{k} G\left(\boldsymbol{\nu}_{\boldsymbol{n}}, k\right)=\frac{\max _{1 \leq i \leq n-1}\left(i-1+\nu_{i}+2 \sum_{j \geq i+1}^{n} \nu_{j}\right)}{\nu_{1}+2 \sum_{j>1}^{n} \nu_{j}}$

Denoting the minimum delay when splitting the packets into $k$ sub-packets by $T_{\text {min }}^{k}$, we can show that,

$$
\lim _{n \rightarrow \infty} \frac{\mathbb{E}\left\{T_{\min }^{k}\right\}}{n}= \begin{cases}2 \mu & \text { if } \mu \geq 1 / 2 k \\ 1 / k & \text { if } \mu \leq 1 / 2 k\end{cases}
$$

Finally assuming a packet erasure channel the impact of noise on data collection time is examined.

Theorem 4. Given a probability $p$ of packet erasure, let $\nu_{i}$ 's be i.i.d. random variables defined before, then:

$$
1 \leq \frac{\mathbb{E}\left(T\left(p, \boldsymbol{\nu}_{\boldsymbol{n}}\right)\right)}{\mathbb{E}\left(T\left(0, \boldsymbol{\nu}_{\boldsymbol{n}}\right)\right)} \leq O(n p)+1
$$

\section{REFERENCES}

[1] P. Gupta and P. R. Kumar, "The capacity of wireless networks," IEEE Transactions on Information Theory, vol. 46, mar 2000.

[2] C. Florens, M. Sharif, R. J. McEliece, "Random Sensory Networks: A Delay Analysis," submitted to IEEE Transactions on Information Theory. 